\title{
Translational positioning of a nucleosomal glucocorticoid response element modulates glucocorticoid receptor affinity
}

\author{
Qiao Li and Örjan Wrange ${ }^{1}$ \\ Department of Cell and Molecular Biology, Medical Nobel Institute, Karolinska Institute, S-171 77 Stockholm, Sweden
}

Positioned nucleosomes are often found in enhancer/promoter regions where they confer defined positioning of trans-active-factor response element(s) relative to the histone octamer. Here, we address how this affects factor/response element recognition. We used 165-bp DNA segments containing one glucocorticoid response element (GRE) and rat liver core histones to reconstitute nucleosomes in vitro. The GREs in these nucleosomes were held in identical helical settings but different translational positions. This was achieved by placing the GRE within or at the flank of a 95-bp DNA-bending sequence. Glucocorticoid receptor (GR)-binding experiments demonstrated that a GRE in free DNA has a 2.5-fold higher affinity for GR than the nucleosomal GRE when positioned 40 bp from the nucleosome dyad. A nucleosomal GRE positioned $40 \mathrm{bp}$ from the nucleosome dyad, on the other hand, binds GR 4.3-fold better as compared to an identical GRE positioned $20 \mathrm{bp}$ from the dyad and 1.4 -fold better than a GRE positioned at the dyad. Interruption of the DNA-bending sequence, by a 5-bp AT segment next to a nucleosomal GRE positioned 20 bp from the dyad, restores GR affinity to the same level as when the GRE is placed $40 \mathrm{bp}$ from the dyad. The effect on GR/GRE affinity either by different positioning within the 95-bp bending sequence or by introducing the 5-bp AT-segment is seen only in a nucleosomal context. We conclude that a translationally positioned nucleosome can modulate the affinity of a trans-active factor for its target response element.

[Key Words: Nucleosome; nucleosome positioning; glucocorticoid receptor; DNA-bending sequence; gene regulation; transcription factor]

Received April 20, 1993; revised version accepted September 16, 1993.

Transcriptional regulation from an inducible gene generally involves the binding of one or several trans-active factors to their cognate DNA-binding sites near the promoter. This protein-DNA recognition may represent the first event in gene induction and may thus be the trigger of this multistep process.

DNA in eukaryotic cells is complexed with histones to form nucleosomes and higher order chromatin structure. An increasing body of evidence suggests that histones and other chromatin-associated factors play an active role in transcriptional regulation. Turning off the synthesis of one core histone in growing yeast cells reduces nucleosome density and concomitantly increases constitutive transcription from several inducible promoters (Han and Grunstein 1988; Durrin et al. 1992). Similar results were obtained with the mouse mammary tumor virus (MMTV) promoter when tested in Xenopus oocytes. Here, the coinjection of MMTV promoter and competitor DNA resulted in reduced nucleosome den-

\footnotetext{
${ }^{1}$ Corresponding author.
}

sity and a corresponding increase of constitutive MMTV transcription (Perlmann and Wrange 1991). Studies on the yeast PHO5 promoter demonstrated that an unstable nucleosome results in increased constitutive expression while a more stable nucleosome reduces inducibility (Straka and Hörz 1991), thus, elegantly demonstrating that histone-DNA interaction makes an intrinsic contribution to the regulation of the PHO5 gene.

The interplay of trans-active factors and chromatin structure is likely to be of functional significance in gene regulation. Detailed structural information of the higher order chromatin structure is not available, but the structure of the core nucleosome has been described in some detail (Richmond et al. 1984; Arents et al. 1991). Nucleosomes can be reconstituted in vitro and used to study nucleosomal DNA structure (Rhodes 1985; Hayes et al. 1991), effects on in vitro transcription (Knezetic and Luse 1986; Lorch et al. 1987, 1992; Losa and Brown 1987; Workman et al. 1988; Clark and Wolffe 1991; Laybourn and Kadonaga 1991), and trans-active factor-DNA interaction (Rhodes 1985; Perlmann and Wrange 1988; Taylor 
et al. 1991; Lee et al. 1993). Here, we extend the studies on how nucleosomal DNA affects trans-active factor/ response element recognition by positioning a single response element in various translational positions while keeping it in an identical rotational setting.

Glucocorticoid receptor (GR)-glucocorticoid response element (GRE) binding is used as a model system because GR induction of the MMTV promoter (RichardFoy and Hager 1987) and the tyrosine amino transferase promoter (Carr and Richard-Foy 1990; Reik et al. 1991) alters the structure of a positioned nucleosome (or nucleosomes/ harboring the GRE in vivo. We and others have shown previously that GR can recognize the MMTV GRE when organized in a positioned nucleosome in vitro (Perlmann and Wrange 1988; Pina et al. 1990). The MMTV GRE, however, consists of a cluster of receptor binding sites with different helical settings arranged in two domains that altogether bind up to four GR homodimers and covers at least $60 \%$ of the DNA in this positioned nucleosome. It is not known to what extent a different rotational or translational position of a nucleosomal GRE will affect GR/GRE recognition. Moreover, we found that cooperative protein-protein interactions between different GR moieties are participating, besides the protein-DNA interactions, in the binding event with free MMTV GRE (Perlmann et al. 1990). Such a complex binding reaction is difficult to dissect functionally. To reduce the complexity of the GR-DNA interaction we designed various nucleosomes harboring a single GRE. Such a GRE is known to interact with one GR homodimer (Tsai et al. 1988; Wrange et al. 1989), which contacts two partially palindromic hexanucleotides and surrounding phosphate groups in two consecutive major grooves on the same side of the DNA double helix (Scheidereit and Beato 1984; Eriksson and Wrange 1990; Luisi et al. 1991). To analyze the effect of different translational positions, we chose to orient the two consecutive major grooves harboring the GRE toward the periphery in a "facing out" configuration on the nucleosome. To achieve this, we exploited the fact that certain synthetic DNA-bending sequences are able to direct the helical setting of DNA on a histone octamer (Shrader and Crothers 1989).

In vitro-binding experiments using purified rat liver GR demonstrate that a GRE in free DNA has a 2.5 -fold higher affinity for GR than the nucleosomal GRE when positioned $40 \mathrm{bp}$ from the dyad. A GRE positioned $40 \mathrm{bp}$ from the dyad, on the other hand, binds GR 4.3-fold better, as compared with an identical GRE positioned $20 \mathrm{bp}$ from the dyad, and 1.4-fold better than the GRE positioned at the dyad. Interruption of the DNA-bending sequence with a 5-bp AT segment beside a GRE positioned 20 bp from the dyad restores GR affinity to the same level as when placed $40 \mathrm{bp}$ from the dyad. The effect on GR/GRE affinity, either by different positioning within the 95-bp bending sequence, or by introducing the 5-bp AT segment, is seen only in a nucleosomal context. Our results show that translational positioning of a nucleosome, and the local DNA sequence flanking the GRE, modulates GR/GRE recognition.

\section{Results}

Defined helical and translational GRE positioning in in vitro reconstituted nucleosomes

A homogeneous and structurally defined nucleosome population must be used to evaluate the effect on GR/ GRE interaction as a function of nucleosome positioning. Shrader and Crothers (1989) demonstrated that repeated $(\mathrm{A} / \mathrm{T})_{3} \mathrm{NN}(\mathrm{G} / \mathrm{C})_{3} \mathrm{NN}$ motifs of DNA preferentially bend around a histone octamer so that $\mathrm{A} / \mathrm{T}$ segments are located at sites of minor groove compression, whereas G/C segments are located at sites of major groove compression. We used one such motif, the TG motif (Shrader and Crothers 1989, Fig. 1), to direct the helical setting of the nucleosomal GRE. A 15-bp GRE,

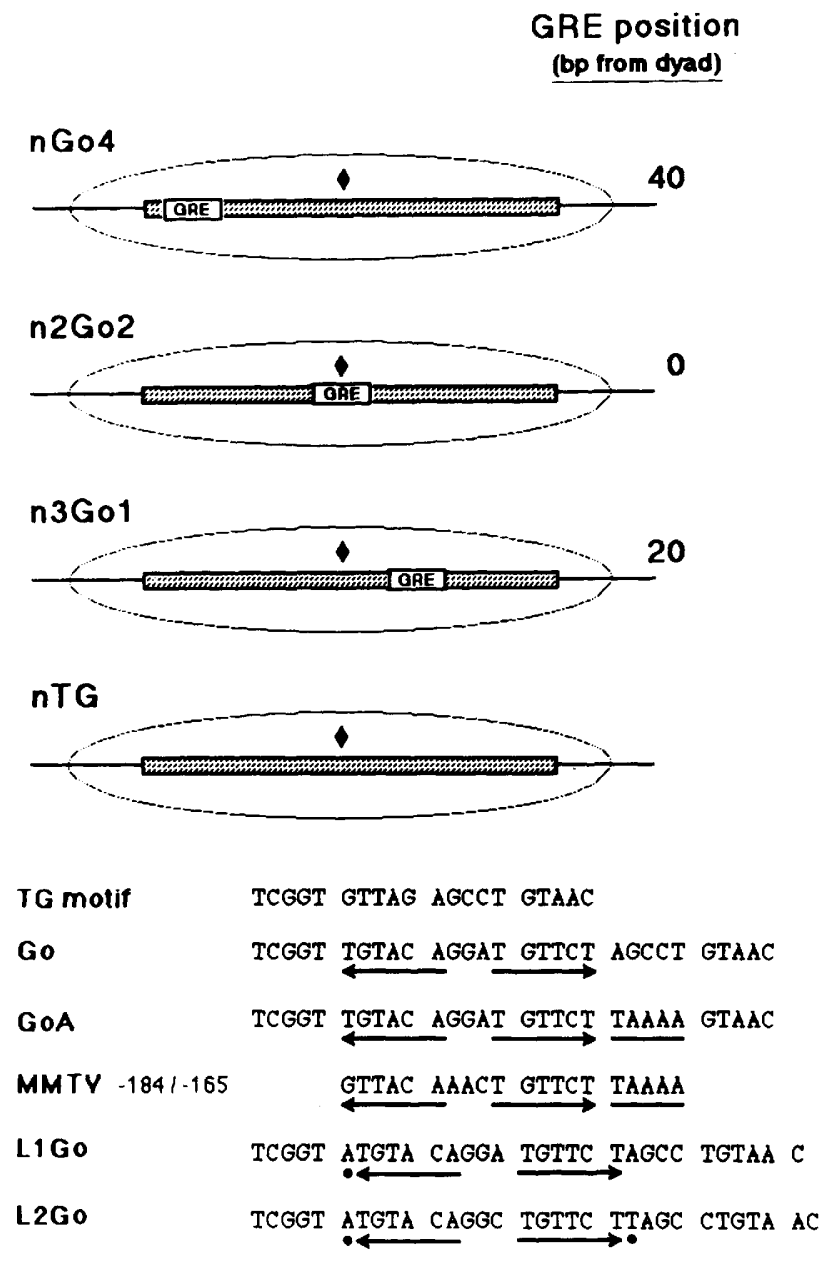

Figure 1. DNA constructs used for in vitro reconstitution of nucleosomes. The translational position of the 15-bp GRE, defined as the number of base pairs from the GRE dyad to the nucleosome dyad, is indicated for each construct. Hatched bars represent the $95 \mathrm{bp}$ of the TG motif (=DNA-bending sequence); solid lines represent vector DNA. ( | Nucleosome dyads. Ellipsoids signify the position of the histone octamer according to exonuclease III protection. Various DNA segments used for construction of artificial nucleosomes are shown below. A GRE from the MMTV promoter is shown for a comparison. 
originating from the $-2509 /-2495$ segment of the tyrosine aminotransferase gene (Jantzen et al. 1987), was placed either within or at the flank of the 95-bp TG motif, so that the two major grooves making contact with GR would be oriented in an outward direction (i.e., away from the center of the histone octamer). The rational for this was to minimize differences in helical setting and to isolate the effects of different GRE translational positions on GR/GRE recognition.

In vitro-reconstituted nucleosomes harboring a positioned GRE, referred to as nGo4, n3Go1, and n2Go2 (Fig. 1), were analyzed by DNase I footprinting, because DNase I preferentially cuts DNA where the minor groove is facing the outside of the nucleosome and thus is bent away from the enzyme cut site (Noll 1974; Simpson 1991). DNase I footprinting of nGo4 showed that both top and bottom strands of reconstituted DNA had an overall 10 -bp periodicity in DNase I sensitivity and protection pattern relative to the free DNA control (Fig. 2A). The cuts on the two strands were staggered about 2-4 bp as expected for DNA arranged in a nucleosome (Fig. 2B). As can be seen, the nucleosomal GRE consensus sequence is oriented with the two major grooves facing the periphery (Fig. 2A,B). The other constructs, n3Go1 and n2Go2 (Fig. 1), showed an identical distribution of DNase I cut sites and, consequently, had the same rotational setting on the nucleosomes as nGo4 (see Fig. 5, below; data not shown).

The translational positioning of nGo4, n3Gol, and n2Go2 was examined by use of exonuclease III protection assay. Exonuclease III DNA digestion is retarded in the presence of histone octamer, as visualized by a histone-induced band pattern with 10 -bp intervals. The first histone-induced stop in nGo4 of both DNA strands de-



B
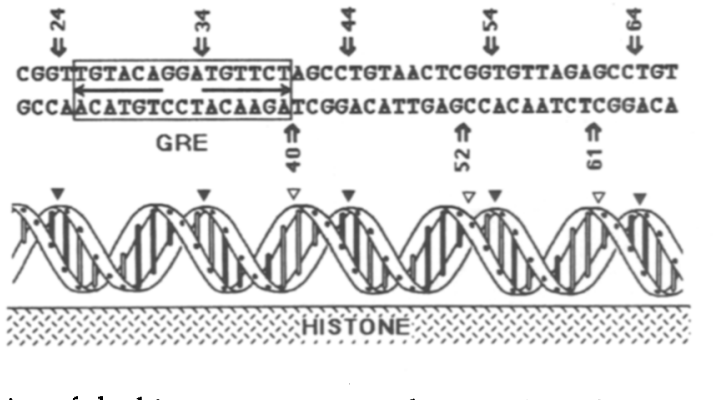

ing of the histone octamer, nucleosome boundaries / solid histone octamer positioning (ellipsoid).
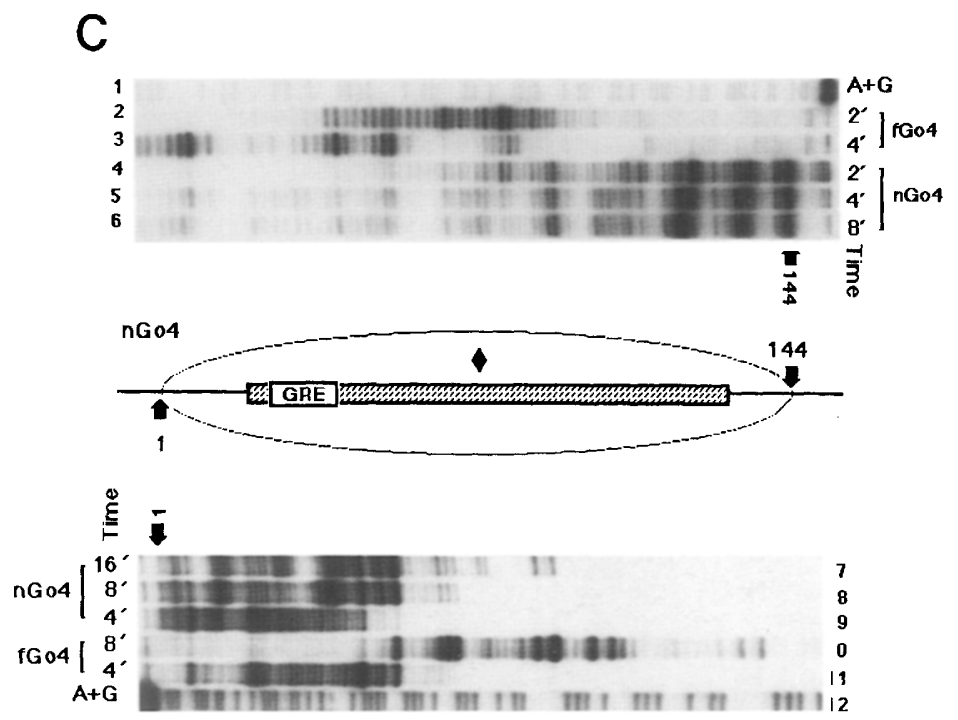

Figure 2. Helical and translational positioning of nGo4. (A) DNase I footprinting of $n$ Go4. Free Go4 DNA fragment ( $(\mathrm{Gro} 4)$ and nGo4 were analyzed with sequencing marker $(A+G)$ for both the bottom strand (lanes 1-5) and the top strand (lanes $6-10$ ) to reveal the helical setting of nGo4. Open arrows show the 10-bp periodic cleavages characteristic of nucleosome-organized DNA. The numbers beside the open arrows are defined by nucleosome boundaries (1-144) with exonuclease III protection. Vertical arrows show GRE position on both strands. $(B)$ Graphic representation of the distribution of DNase I cuts in nGo4. The helical setting of the DNA on the histone octamer is illustrated as revealed by the DNase I cut sites on both the bottom strand $(\nabla)$ and the top strand $(\nabla)$. The GRE segment is enclosed in a box; the horizontal arrows between the two DNA strands signify the partial palindrome that is recognized by GR in two consecutive major grooves. These major grooves are exposed (i.e., facing the periphery) as seen in the DNA diagram. $(C)$ Exonuclease III protection analysis of nGo4. The first exonuclease III stops on both the top (lanes 1-6) and the bottom (lanes 7-12) strands are indicated by solid arrows. There are $144 \mathrm{bp}$ of DNA between the arrows. Free Go4 (fGo4) was analyzed in parallel with nGo4 as a control for sequence-specific exonuclease III stops. Exonuclease III digestion times are given in minutes as indicated. A $+\mathrm{G}$ marks the sequencing ladder. The middle panel represents a diagrammatic summary of the translational positionarrows), dyad ( $\mid$ ), TG motifs (hatched bar), location of GRE (open bar), and 
fined a 144-bp DNA segment (Fig. 2C). The size of the protected DNA fragment and the pattern of exonuclease III digestion agree with DNA being organized in a core nucleosome (Ramsay 1986). The 144 bp of exonuclease III-protected nGo4 DNA segment, in combination with the absence of any histone-induced exonuclease III protection at the DNA ends, is compatible with one strongly preferred translational position of the DNA fragment on the histone octamer. We cannot exclude that a minor fraction of alternatively positioned nucleosomes are present in our reconstituted nucleosome preparations. We were unable, however, to detect any alternative nucleosome positioning even after strong overexposure of several exonuclease III experiments, which infers that alternatively positioned nucleosomes, if present, will not significantly influence our binding data. The two other constructs harboring a nucleosomal GRE, n3Go1, and n2Go2 (Fig. 1), had the first histone-induced exonuclease III stop on either strand at identical positions as shown for nGo4, thus confirming an identical translational positioning of the 165-bp DNA fragment on the histone octamer irrespective of the GRE position (data not shown). The distances between the central base pairs of the partially palindromic GRE and the nucleosome dyad, as defined by exonuclease III protection, were 40,20 , and 0 bp for $\mathrm{nGo} 4, \mathrm{n} 3 \mathrm{Gol}$, and $\mathrm{n} 2 \mathrm{Go} 2$, respectively (Fig. 1).

We conclude that nGo4, n3Gol, and n2Go2 show identical helical settings and have the same translational positioning of the DNA fragment relative to the histone octamer.

\section{Translational nucleosome positioning modulates GR-} GRE interaction

The three nucleosomes harboring a differently positioned GRE, nGo4, n3Go1, and n2Go2, were assayed for GR-binding affinity by mobility retardation assay. A nucleosome construct harboring 5 repeats of the 20-bp TG motif but lacking a GRE (nTG, Fig. 1) was used to measure nonspecific binding, which was subtracted from the total binding of GR to the GRE-containing mononucleosomes to determine the specific GR binding. In these experiments, GR concentration was kept constant while nucleosome concentration was varied. The binding reactions were conducted at a GR concentration of $<1.3 \mathrm{~nm}$ to assure that the complex formation is driven by GR/ GRE affinity and to minimize nonspecific binding and aggregation. Similar problems of concentration-dependent GR aggregation and high nonspecific binding in mobility retardation experiments have been observed previously (Eriksson and Wrange 1990).

Figure $3 \mathrm{~A}$ shows a representative experiment in which GR concentration was kept constant at $1.2 \mathrm{nM}$, while nucleosome concentration was varied from 19 to $75 \mathrm{pm}$. Comparison of the relative GR-binding affinity for $n \mathrm{Go} 4$, $\mathrm{n} 3 \mathrm{Gol}$, and $\mathrm{n} 2 \mathrm{Go} 2$ (Fig. 3B) revealed distinct differences. At 75 pM nucleosome concentration, the specific GR complex formation with $\mathrm{nGo} 4$ was 4.3 -fold higher as compared with n3Go1. In contrast, nGo4 had only a 1.4- fold higher GR complex formation when compared with $\mathrm{n} 2 \mathrm{Go} 2$. The same relative difference in complex formation was observed over a wide range of nucleosome concentrations (data not shown). There was no detectable difference in GR affinity between Go4, 3Gol, and 2Go2 in the free state (i.e., in the absence of histones) when analyzed at a GR concentration of $0.3 \mathrm{nM}$, which is equivalent to the apparent dissociation constant $\left(K_{\mathrm{d}}\right)$ of GR binding to the MMTV GRE (Perlmann et al. 1990).

Free GRE DNA formed 2.5 \pm 0.6 -fold $(n=5)$ more GR complex than the best of the nucleosomal GREs (i.e., $\mathrm{nGo4}$ ) in the same experimental setup as in Figure 3A but using a GR concentration of $0.6 \mathrm{nM}$ (data not shown). This is in agreement with previous work concerning the upstream GR-binding region of the MMTV, which showed a 2.3-fold higher GR affinity for the free GRE than the nucleosomal G.RE (Perlmann 1992).

Calf thymus DNA (CT DNA) competition experiments, in which the concentration of the different nucleosomal GREs and GR was kept constant in the presence of increasing concentrations of CT DNA competitor, also demonstrated a similar difference in relative GR affinity as above (Fig. 3C). That the retarded GR-nucleosome complex resulted from sequence-specific GR interaction was confirmed by methylation interference experiments of nGo4 (Fig. 3D). This revealed the same pattern of methylation interference as seen for free GRE (Eriksson and Wrange 1990; Q. Li and Ö. Wrange unpubl.). This experiment also demonstrated that a decreased concentration of CT DNA competitor results in a larger fraction of norispecific GR-nucleosome complex, appearing as a less distinct pattern of methylation interference [Fig. 3D, cf. lane $3(0.8 \mu \mathrm{g} / \mathrm{ml}$ CT DNA) with lanes 4 and $5(0.2 \mu \mathrm{g} / \mathrm{ml}$ CT DNA $)]$. To avoid the contribution of such nonspecific GR binding, we used $0.8 \mu \mathrm{g} / \mathrm{ml}$ of CT DNA as the starting point in these competition experiments (Fig. 3E). That this concentration of CT DNA eliminated nonspecific binding was also confirmed by the absence of any retarded complex in the nTG control (data not shown). At $12.8 \mu \mathrm{g} / \mathrm{ml}$ of CT DNA competitor, the concentration of GR binding for nGo4 was $22.7 \pm 7.6 \%(n=9)$ of control. The corresponding level of binding for $n 3 \mathrm{Gol}$, and $n 2 \mathrm{Go} 2$ was $3.4 \pm 3.1 \%$ $(n=7)$ and $14.5 \pm 3.4 \% \quad(n=6)$, respectively (Fig. 3E). Again, no difference in GR/GRE affinity was detected when the corresponding free DNA fragments were used in CT DNA competition experiments (Table 1).

We conclude that there is a distinct difference in relative GR affinity for a nucleosomal GRE when placed in different translational positions within the nucleosome. Moreover, the relative difference in GR affinity for a free GRE and a nucleosomal GRE for the construct Go4 is smaller than the difference between two differently positioned nucleosomal GREs, such as nGo4 and n3Gol.

A TAAAA DNA segment beside the nucleosomal GRE in $3 G o 1$ and $2 G 02$ restores $G R$ affinity

To evaluate the role of the DNA-bending sequence in the position dependence of GR/GRE interaction, we re- 

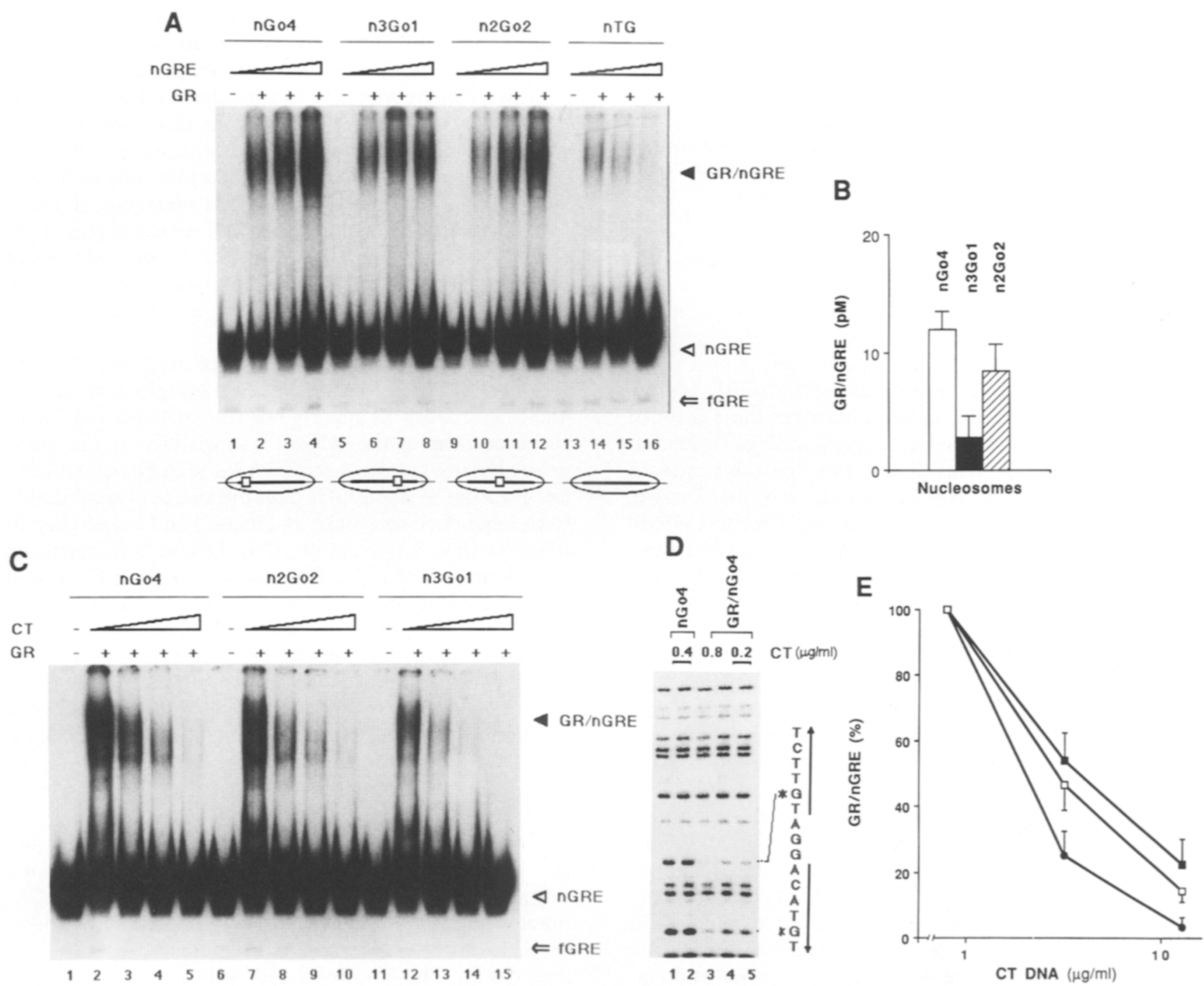

Figure 3. Effect of GRE translational positions on GR affinity. (A) Relative affinity assayed by nucleosome titration. The mobility retardation experiment was carried out in the presence $(+)$ or the absence (-) of GR (1.2 nM), with increasing nucleosome concentrations $(18.8,37.5$, and $75.0 \mathrm{pM}$ ) of nGo4 (lanes 1-4), n3Gol (lanes 5-8), or n2Go2 (lanes 9-12). nTG (lanes 13-16) was included as a negative control to subtract nonspecific GR binding for each nucleosome concentration, the obtained difference thus representing specific GR/nGRE binding. ( $\Delta$ ) Retarded complexes of GR and nucleosomal GRE (GR/nGRE); $(\triangle)$ unbound nucleosomes. The open arrow shows the position of free DNA, only visible after prolonged exposure. The positions of the GRE in different nucleosomes are shown by the illustrations below their respective lanes. ( $\square$ ) GRE; (ellipsoids) histone octamer; (solid lines) TG motif. (B) A graphic comparison of nucleosome titration experiments. The relative GR affinity was expressed as concentration of specific nucleosome-GR complex at a nucleosome concentration of $75 \mathrm{pM}$ for $\mathrm{nGo} 4(11.9 \pm 1.5 \mathrm{pM}, n=7)$, n3Gol $(2.8 \pm 1.7 \mathrm{pM}, n=8)$, and $\mathrm{n} 2 \mathrm{Go} 2(8.4 \pm 2.1 \mathrm{pM}$, $n=6$ ) (for definition of specific binding, see legend to $A$ ). (C) Relative affinity assayed by CT DNA competition. The GR/nGRE complex was analyzed as a function of increasing amounts of CT DNA competitor (CT) at concentrations $0,0.2,0.8,3.2$, and $12.8 \mu \mathrm{g} / \mathrm{ml}$ for nGo4 (lanes 1-5), n2Go2 (lanes 6-10), and n3Gol (lanes 11-15). Lanes + or - denote the presence or the absence, respectively, of GR $(3.6 \mathrm{nM})$ in the reaction. ( $\Delta)$ The retarded complex of GR and nucleosomal GRE /GR/nGRE); $(\triangle)$ unbound nucleosomes. The open arrow shows the position of free DNA, only visible after prolonged exposure. $(D)$ Specific binding evaluated by methylation interference. The GR/nGo4 complex (lanes 3-5) and the unbound nGo4 (lanes 1,2) were cut out from the same gel lanes in the experiment performed as in $C$, isolated by transfer to and elution from a DEAE membrane (Schleicher \& Schuell), followed by denaturing sequencing gel analysis and PhosphorImager evaluation. A distinct methylation interference of two guanine residues $\left({ }^{*}\right)$ is seen in lane 3 in the presence of $0.8 \mu \mathrm{g} / \mathrm{ml}$ of competitor CT DNA; a less pronounced interference pattern is seen when the CT DNA is decreased to 0.2 $\mu \mathrm{g} / \mathrm{ml}($ lanes 4,5$)$. (E) Graphic representation of CT DNA competition. Experiments performed as in $C$ were quantified for nGo4 (a, $n=9)$, n2Go2 $(0, n=7)$, and $\mathrm{n} 3 \mathrm{Gol}(\square,(n=6)$. Specific binding $(100 \%)$ was set at $0.8 \mu \mathrm{g} / \mathrm{ml}$ of CT DNA according to methylation interference experiments and the nTG control, which demonstrated that this CT DNA concentration was required to avoid contribution by nonspecific binding. 
Table 1. CT DNA competition for free DNA

\begin{tabular}{lccc}
\hline & \multicolumn{2}{c}{ GR/fGRE $\{\%\}$} & \\
\cline { 2 - 3 } Free DNA & $3.2 \mu \mathrm{g} / \mathrm{ml} \mathrm{CT}$ & $12.8 \mu \mathrm{g} / \mathrm{ml} \mathrm{CT}$ & Number \\
\hline fGo4 & $51.8 \pm 12.2$ & $18.7 \pm 8.3$ & 11 \\
f2Go2 & $48.8 \pm 9.5$ & $20.6 \pm 1.8$ & 4 \\
f3Go1 & $47.9 \pm 8.3$ & $25.4 \pm 8.9$ & 4 \\
\hline
\end{tabular}

Experiments were done at a GR concentration of $0.9 \mathrm{nM}$ and probe concentration of $75 \mathrm{pm}$. Figures given in the table are calculated as percent of remaining GR/nucleosome complex at the given concentration of CT DNA competitor $(\mathrm{CT})$. Binding at $100 \%$ is the amount of complex present at $0.8 \mu \mathrm{g} / \mathrm{ml}$ of CT DNA that was required to eliminate nonspecific binding (Fig. $4 \mathrm{D}$; data not shown).

placed a AGCCT segment flanking the GRE with a TAAAA segment. This change interrupts the TG motif at the site of major groove compression (Fig. 1). The rationale for this particular design was that the upstream GR-binding region of MMTV has an identical flanking sequence (Fig. 1) and that Shrader and Crothers (1990) showed the flexibility of a AAAAA tract to be anisotropic and detrimental to nucleosome formation when positioned at a site of major groove compression. We reasoned that similar effects might be obtained with a TAAAA segment, which occurs naturally in MMTV GRE. These constructs were assembled on histone octamers to form the nucleosome probes n3GoAl and $\mathrm{n} 2 \mathrm{GoA} 2$ and were compared in GR binding experiments with their corresponding nucleosome constructs n3Gol, and n2Go2, lacking the TAAAA segment. Figure 4, A and $B$, illustrates a nucleosome titration experiment in which GR concentration was kept constant at $1.2 \mathrm{~nm}$ while nucleosome concentration was varied from 9 to $112 \mathrm{pM}$, thus, comparing GR affinity for $\mathrm{n} 3 \mathrm{Gol}$, and $\mathrm{n} 3 \mathrm{GoAl}$. The result shows that a pronounced increase in GR affinity is obtained by the introduction of the TAAAA segment. Figure $4 \mathrm{C}$ summarizes a series of experiments comparing the specific GR binding of n3GoAl and $\mathrm{n} 2 \mathrm{GoA} 2$ with $\mathrm{nGo} 4$ at $75 \mathrm{pM}$ concentration of nucleosome probe and demonstrates that introduction of the TAAAA segment restores GR affinity to the same level as seen in nGo4. CT DNA competition experiments also confirmed that $\mathrm{n} 3 \mathrm{GoA} 1$ and $\mathrm{n} 2 \mathrm{GoA} 2 \mathrm{had}$ relative GR affinity similar to nGo4 (Table 2). If, on the other hand, free DNA was used as probe, there was no detectable effect on GR affinity by introducing the TAAAA segment either by analysis in the presence of increasing amounts of DNA probe (data not shown) or by competition with CT DNA (Fig. 4D).

DNase I footprinting was used to study the effect of the TAAAA segment on the histone-DNA contacts in the nucleosome. These experiments showed that the downstream boundary of n3GoA1 (i.e., downstream of the TAAAA motif) lacked the histone-induced DNase I pattern that was present in the corresponding control (i.e., n3Gol) (Fig. 5A,B; note that Fig. 5B is a lighter exposure of the experiment shown in $5 \mathrm{~A}$, where the lanes in the presence of GR have been deleted). By comparison of lanes 6 and 7 with lanes 15 and 16, it is evident that the nucleosome-induced DNase I cut seen at position 124 in n3Gol is not present in n3GoA1. This demonstrates that the TAAAA motif has disturbed the DNA organization within the downstream TG motif of n3GoA1. Furthermore, the exonuclease III-specific stop on both strands of n3GoAl was at the same position when compared with n3Gol, but the histone-induced exonuclease III pattern was less distinct on the $3^{\prime}$ side [i.e., close to the TAAAA segment (data not shown)]. This supports the notion that the TAAAA segment interferes with the surrounding histone-DNA contacts but not the translational positioning. n2GoA2 showed the identical exonuclease III pattern as its counterpart n2Go2 (data not shown).

There was no qualitative difference in DNase I pattern when comparing $n 2 \mathrm{Go} 2$ and n2GoA2 (Fig. 5C, cf. lanes 6 and 7 with lanes 15 and 16, position 104 and 124), with the exception of the DNase I sensitivity at the compressed major groove of the TAAAA segment in $\mathrm{n} 2 \mathrm{GoA} 2$ (see position 84 in Fig. 5C). This difference is attributable to the sequence specificity of DNase I and is also seen in n3GoAl (Fig. 5A, position 104). DNase I footprinting also demonstrated the GR-induced protection, which was located at the GRE both in free DNA and in its nucleosomal counterpart (Fig. 5A,C). The higher GR affinity to $\mathrm{n} 3 \mathrm{GoA} 1$ as compared with $\mathrm{n} 3 \mathrm{Gol}$ is evident (Fig. 5A; cf. GR-induced footprints in lanes 8 and 9 with lanes 17 and 18), but the quantitative difference is better evaluated by gel retardation assay (Fig. 4A,B). GR-induced DNase I footprints are also seen at the nucleoso$\mathrm{mal}$ GRE in $\mathrm{n} 2 \mathrm{GoA} 2$ and $\mathrm{n} 2 \mathrm{Go} 2$ (Fig. 5C, lanes $8,9,17,18)$.

The experiments in Figure 4 show that the introduction of a TAAAA tract near the GRE allows it to adopt a more open configuration as revealed by a higher GRbinding affinity. This is caused by decreased or disordered histone-DNA contacts as indicated for n3GoAl (Fig. 5A,B). The improved GR affinity caused by the TAAAA tract is only detectable when the GRE is in a nucleosomal context.

\section{An increase in base pairs per helical turn around the GRE does not improve GR affinity}

Quantitative hydroxyl radical footprinting experiments have demonstrated that the three central periods of DNA helix in the positioned nucleosome of the 5S RNA gene from Xenopus borealis have a 10.7-bp helical periodicity, in contrast to the rest of the nucleosome, which has only a 10.0-bp periodicity (Hayes et al. 1991). The TG motif used in our constructs has an overall periodicity of 10.0 bp (Fig. 2A; Shrader and Crothers 1989). This might result in local overtwisting of DNA, especially near the nucleosome dyad, which in turn could be the reason for a decreased GR affinity in $\mathrm{n} 3 \mathrm{Gol}$ and $\mathrm{n} 2 \mathrm{Go} 2$. To address this, we introduced either one or two additional base pairs immediately before or before and after the GRE in n3Gol and n2Go2 (see L1Go and L2Go in Fig. 1) to re- 

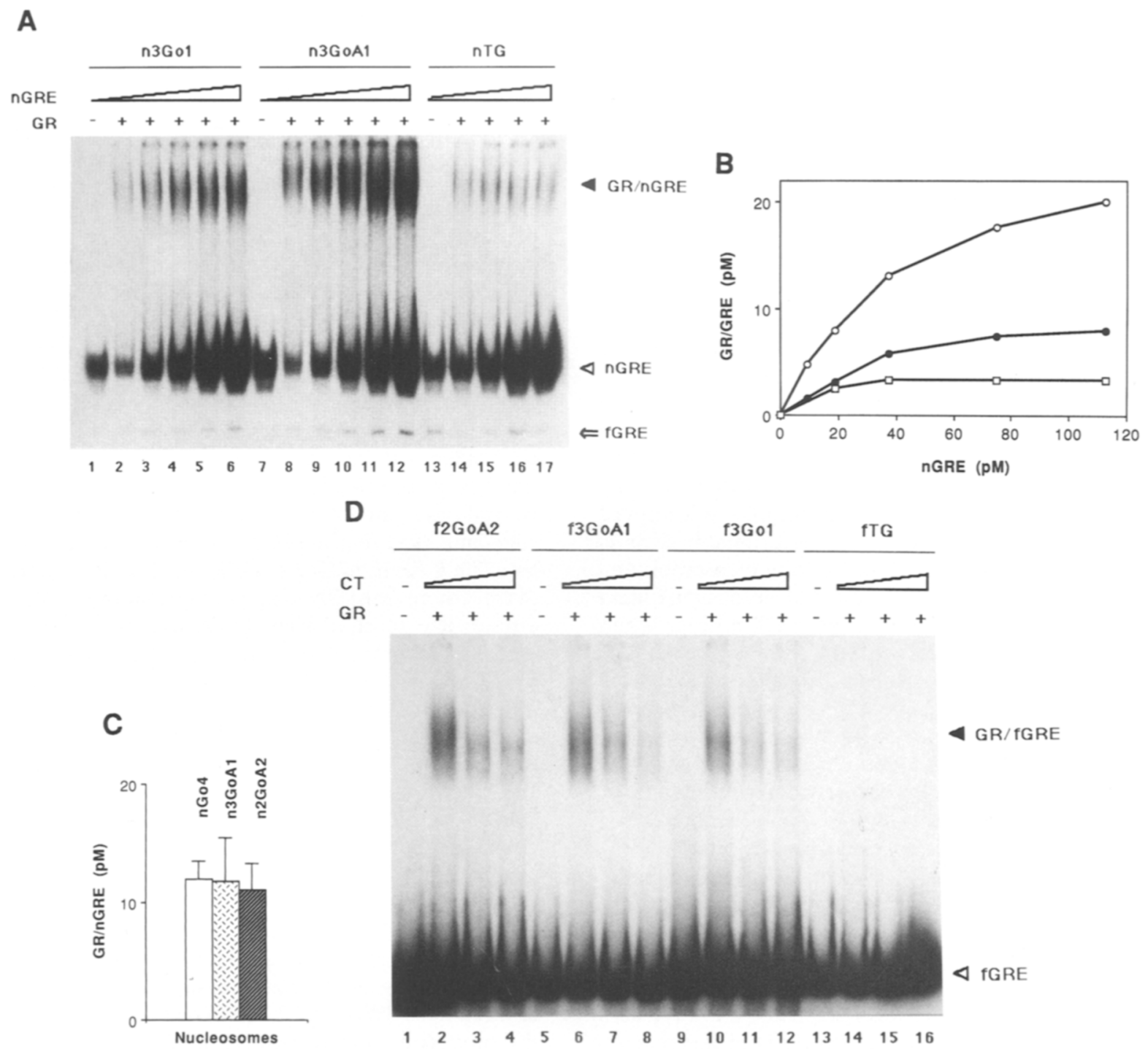

Figure 4. Influence of a GRE-flanking TAAAA segment on GR affinity in n3GoAl and n2GoA2. (A) Relative affinity of n3GoA1 assayed by nucleosome titration. The relative GR affinity of $n 3$ Gol (lanes 1-6) and $n 3 G o A l$ (lanes $7-12$ ) was analyzed by incubating a constant concentration of GR $(1.2 \mathrm{nM})$ with increasing concentrations of the indicated nucleosome probe $(9.4,18.8,37.5,75.0$, and 112.5 pM). nTG (lanes 13-17) was used as control for nonspecific GR binding. (+ or - | The presence or the absence, respectively, of GR in the reaction. ( $(\Delta)$ Specific GR/nGRE complex. $(\Delta)$ Unbound nucleosomes. The open arrow shows the position of free DNA visible only after prolonged exposure. $(B)$ Graphic representation of the experiment in $A$. $(O)$ n3Gol; $(O)$ n3GoAl; (D) nTG. (C) Graphic comparison of nucleosome titration experiments of $\mathrm{n} 3 \mathrm{GoAl}$ and $\mathrm{n} 2 \mathrm{GoA} 2$. The binding of $\mathrm{GR}$ to $\mathrm{n} 3 \mathrm{GoA} 1$ and $\mathrm{n} 2 \mathrm{GoA} 2 \mathrm{was}$ compared with nGo4 by quantification of the specific GR/nGRE complex as in $A$. The concentration of GR/nGRE complex at 75 pM concentration of nucleosome probe $n G o 4, n 3 G o A l$, and $n 2 \mathrm{GoA} 2$ was $11.9 \pm 1.5 \mathrm{pM}(n=7), 11.8 \pm 3.4 \mathrm{pM}(n=8)$, and $11.0 \pm 2.1 \mathrm{pM}(n=8)$, respectively. $(D)$ Relative GR affinity for free GRE assayed by CT DNA competition. The binding of GR to f2GoA2 (lanes $1-4)$ and f3GoAl (lanes 5-8) was analyzed in the presence of increasing amounts $(0,0.8,3.2$, and $12.8 \mu \mathrm{g} / \mathrm{ml}$ ) of CT DNA. f3Gol (lanes 9-12) was included as a comparison, and fTG (lanes 13-16) was employed ds a negative control. GR concentration and probe concentration were kept constant at 0.9 and $75 \mathrm{pM}$, respectively. At $3.2 \mu \mathrm{g} / \mathrm{ml}$ of CT DNA, the remaining GR/GRE complex for f2GoA2, f3GoA1 and f3Gol was $48.1 \pm 11.3 \%(n=4), 55.7 \pm 3.5 \%(n=3)$, and $47.9 \pm 8.3 \%(n=4)$, respectively.

lieve any possible overtwist. DNase I footprinting of such nucleosomes demonstrated that the helical 10-bp periodicity of preferred DNase I cut sites in the flanking TG motif involved the same phosphodiester bonds as in the original constructs $\mathrm{n} 3 \mathrm{Go1}$ and $\mathrm{n} 2 \mathrm{Go} 2$ /data not shown). This suggests that the one to two extra base pairs near the GRE did result in a local periodicity shift, which was accommodated around the nucleosomal GRE and did not spread to the TG motif. It thus resulted in a shift in local periodicity around the nucleosomal GRE of 10.7 and 11.3 bp per helical turn for the L1Go and L2Go constructs, respectively. In addition, the DNase I foot- 
Table 2. CT DNA competition for nucleosomal DNA

\begin{tabular}{lccc}
\hline \multirow{2}{*}{$\begin{array}{l}\text { Nucleosomal } \\
\text { DNA }\end{array}$} & \multicolumn{2}{c}{ GR/nGRE (\%) } & \\
\cline { 2 - 3 } & $3.2 \mu \mathrm{g} / \mathrm{ml} \mathrm{CT}$ & $12.8 \mu \mathrm{g} / \mathrm{ml} \mathrm{CT}$ & Number \\
\hline n3GoA1 & $56.3 \pm 10.6$ & $25.4 \pm 7.6$ & 5 \\
n2GoA2 & $56.2 \pm 10.3$ & $23.9 \pm 7.6$ & 7 \\
nGo4 & $54.3 \pm 7.8$ & $22.7 \pm 7.0$ & 9 \\
\hline
\end{tabular}

Experiments were done at a GR concentration of $3.6 \mathrm{nM}$. Other conditions are as in Table 1.

printing demonstrated that the helical setting of the GRE in the L2Go constructs are facing the periphery as seen for n3Gol and n2Go2. The L1Go constructs are expected to have a slightly altered helical setting because of the asymmetric addition of $1 \mathrm{bp}$ on the $5^{\prime}$ side of the GRE. Exonuclease III digestion demonstrated that the first histone-induced stop for both strands was moved $\sim 1 \mathrm{bp}$ toward the center when two additional base pairs has been added near the GRE in L2Go nucleosomes [i.e., these nucleosomes still organized 144 bp of DNA /data not shown)]. There was no change in GR affinity when comparing such constructs with $\mathrm{n} 3 \mathrm{Gol}$ and $\mathrm{n} 2 \mathrm{Go}$. Furthermore, free DNA showed no difference in GR/GRE affinity for any of these constructs when comparing them with 3Gol and 2Go2, which is not surprising because the GRE-binding sequence remained unchanged. We conclude that the observed GR affinity difference for different translational positions of a nucleosomal GRE is not attributable to the overtwisting of the DNA.

\section{Discussion}

Our results show that a GRE organized in a nucleosome has different affinities for GR depending on the translational positioning and the local sequence context. This relative difference in GR/GRE affinity is of larger magnitude when comparing different translational positioning within a nucleosome than the difference observed when comparing the nucleosomal GRE with the highest GR affinity (i.e., nGo4) with an identical GRE in histonefree DNA.

The center of the 15-bp GRE in nGo4 is positioned 40 bp from the nucleosome dyad. According to structural studies, this infers that the GRE in nGo4 is located at the domain of histone H2B and histone H4 (Richmond et al. 1984; Arents et al. 1991). In addition, the GRE in nGo4 is positioned at one end of the TG motif (Fig. 1), and the histone-induced DNase I pattern of nGo4 does not include any cut sites distal to the GRE. This implicates that the GRE in nGo4 is associated more loosely with the histone octamer on its distal side. This is in marked contrast to the GRE positions in n3Gol and n2Go2, which are both flanked by $20-60$ bp of TG motif causing a well-ordered bend around the histone octamer as shown by a distinct histone-induced DNase I pattern of $\sim 10$-bp periodicity on both sides of the GRE (Fig. 5A,C). That the flanking DNA sequence is an important determinant for GR-GRE affinity within a nucleosome is demonstrated by the improved GR affinity after exchanging a 5-bp AGCCT bending sequence with a 5-bp TAAAA segment as in $\mathrm{n} 3 \mathrm{GoA} 1$ (Figs. 1 and 4). This suggests that the flanking DNA sequence (i.e., the lack of TG motif on the distal side of the GRE) probably contributes to the relatively high affinity for GR in the nGo4. Whether the local histone-GRE contacts at this site contribute to the high GR affinity remains to be determined (e.g., by constructs where the GRE is engulfed also on the distal side by a longer segment of DNA-bending sequence).

The difference in GR affinity for $\mathrm{n} 3 \mathrm{Gol}$ and $\mathrm{n} 2 \mathrm{Go} 2$ is probably caused by different translational positioning within the nucleosome because the flanking DNA sequence surrounding these GREs is identical for at least $20 \mathrm{bp}$ in either direction and because both constructs have identical helical settings with the two major grooves of the GRE facing the periphery. n3Gol has the central base pairs of its GRE located $20 \mathrm{bp}$ from the nucleosome dyad (Fig. 1), which is in the region of histone $\mathrm{H} 4$. The GRE in $\mathrm{n} 2 \mathrm{Go} 2$ is positioned at the nucleosome dyad, where the DNA is contacting the histone $\mathrm{H} 3$ dimer. The local histone-DNA contacts might cause sterical hindrance resulting in the difference in GR affinity for $\mathrm{n} 3 \mathrm{Gol}$ and $\mathrm{n} 2 \mathrm{Go2}$. The low GR affinity for n3Gol, however, might also be attributable to the previously described bend, or kink, in nucleosomal DNA that occurs $\sim 1$ (Richmond et al. 1984) or 1.5 (Hogan et al. 1987) helical turns from the nucleosome dyad. This anisotropic kink in DNA might distort the proximal major groove of the GRE, which is located 1.5 turns from the dyad in $\mathrm{n} 3 \mathrm{Gol}$ and, hence, partially inhibit GR binding.

Is a 4.3-fold difference in GR/GRE affinity caused by different translational positioning of enough magnitude to play a role in transcriptional regulation? Studies in yeast have suggested that a group of proteins, usually referred to as SWI1, SWI2, and SWI3, may be involved in the process of transcriptional induction of certain promoters by cooperating with trans-active factors. Genetic and biochemical evidence suggests that the SWI1, SWI2, and SWI3 gene products act by modifying or opening up chromatin (Hirschhorn et al. 1992; Peterson and Herskowitz 1992; Winston and Carlson 1992). Experiments involving the introduction of GR in yeast have demonstrated that GR-mediated gene induction in vivo requires functional SWI1, SWI2, and SWI3 gene products (Yoshinaga et al 1992). One might speculate that a transactive factor functions by directing a chromatin opening activity to the site of action. Whether the SWII, SWI2, and SWI3 products serve such a function remains to be shown. If GR acts by targeting a chromatin opening activity to a gene segment surrounding a GRE, then even small differences in GR/GRE affinity would be expected to correlate to the efficiency to activate a promoter.

Translational positioning could be used in the living cell to construct hierarchies of factor occupancy. According to our data, two alternative situations are apparent for a trans-active factor like GR (Fig. 6). In gene A, a GRE, positioned in an open nucleosome context may act as a primary factor and trigger activation, perhaps by 


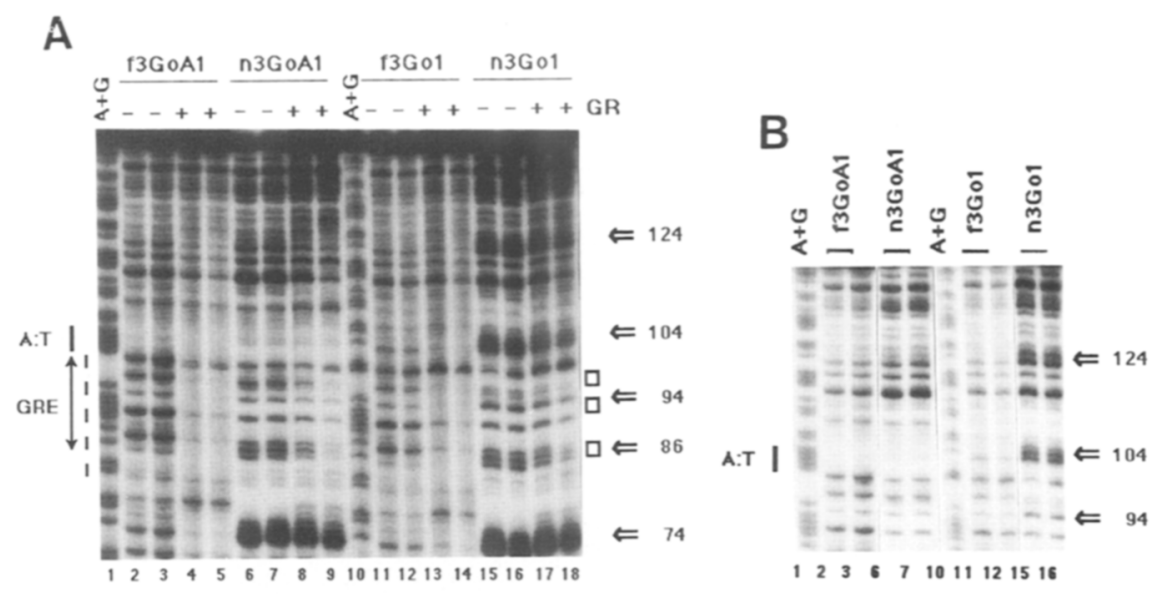

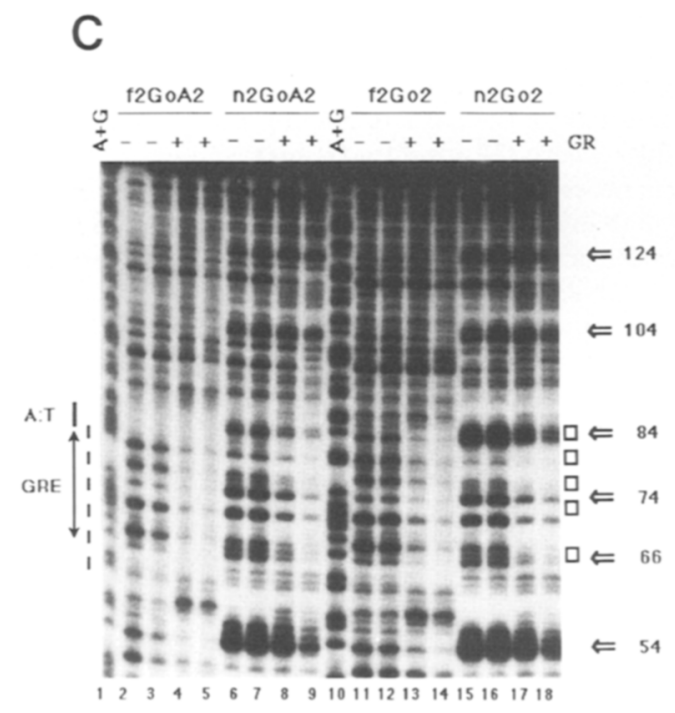

Figure 5. Effects on histone-DNA contacts by introducing a flanking TAAAA segment near the GRE. (A) DNase I footprinting of the top strand of n3GoAl and n3Gol. Free DNA 3GoAl (f3GoAl, lanes 2-5) and 3Gol (f3Gol, lanes 11-14) were analyzed in parallel with n3GoA1 (lanes 6-9) and n3Gol (lanes 15-18). $\mathrm{A}+\mathrm{G}$ marks the sequencing ladder. (+ or -$)$ The presence or the absence, respectively, of GR at concentrations of $1.8 \mathrm{~nm}$ (lanes 4,13), $3.6 \mathrm{nM}$ (lanes $5,8,14,17)$ and $7.2 \mathrm{nM}$ (lanes 9,18). Open arrows show the periodic cleavages of $\sim 10$ bp characteristic for nucleosome-organized DNA. The numbers beside the open arrows are defined according to exonuclease III analysis of nucleosome boundaries. The vertical arrow shows the GRE position; the vertical line denotes the AT tract; the broken line and open squares indicate GR-induced DNase I protection in free GRE and nucleosomal GRE, respectively. $(B)$ A lighter exposure of the upper segment of $A$ with deletion of lanes in the presence of GR. This illustrates the absence of a histone-induced DNase I cut at position 124 in n3GoAl as opposed to the histone-induced cut seen at this position in $\mathrm{n} 3 \mathrm{Gol}$. Lane numbers are as in $A$. $(C)$ DNase I footprinting of the top strand of n2GoA2 and $\mathrm{n} 2 \mathrm{Go} 2$ (the same experimental design as in $A$ ). The corresponding free DNAs (f2GoA2 and f2Go2) were analyzed in parallel. All symbols are as in $A$. chromatin unfolding, whereas an adjacent factor response element (XRE), which binds an arbitrary factor $\mathrm{X}$, is positioned in a closed nucleosome context and will only be occupied after chromatin unfolding has been triggered by the primary factor. Factor $\mathrm{X}$ thus represents a secondary factor in this hierarchy. In gene $B$, the opposite arrangement has occurred, with the GRE positioned in a closed nucleosome context and the XRE in an open configuration, thus reversing the binding hierarchy when compared with gene $\mathrm{A}$. In this case, factor $\mathrm{X}$ would trigger chromatin unfolding, which then allows the binding of GR. The MMTV promoter represents an example of a factor-binding hierarchy similar to the gene A model. Glucocorticoids are known to trigger a transition of a positioned nucleosome into a more open chromatin structure (Zaret and Yamamoto 1984; Richard-Foy and Hager 1987; Bresnick et al. 19921, which then results in the binding of nuclear factor-1 (NF-1) to its response element located within the same nucleosome as the GRE (Cordingley et al. 1987). We are not aware of a naturally occurring gene B model involving GR. Our results, however, show that by taking advantage of nucleosome positioning, one single factor may acquire the potential to concomitantly act as a primary factor in one gene and as a secondary factor in another, thus allowing differential modes of regulation of two separate genes in the same cell (Fig. 6). In this context, a positioned nucleosome may be regarded as a higher order modulator for sequence-specific trans-active factor recognition or even as an intergrated part of the various DNA-protein acceptor sites designed for binding of trans-active factors and thereby confer gene regulation.

Our studies show that a GRE within different nucleosomal contexts modulates its accessibility for GR binding in vitro. The functional consequences of GRE translational positioning must be evaluated in vivo to test the model described above. It is already known, however, that histone-DNA contacts may have intrinsic contributions to the regulation of an inducible promoter (Straka and Hörz 1991) and that changing small segments or even a single amino acid within certain histones may have selective effects on the transcriptional regulation of certain genes (Johnson et al. 1990; Durrin et al. 1991). Furthermore, the presence of positioned nucleosomes has been shown to affect other cellular processes, for example, to inhibit replication (Simpson 1990) 


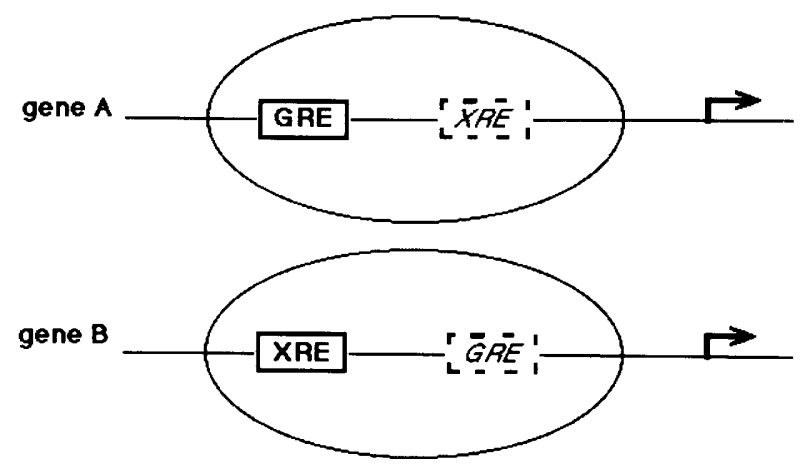

Figure 6. A model for two alternative binding hierarchies. The model involves a GRE and a response element for an arbitrary factor X (XRE). The ellipsoid signifies a nucleosome, the solid line represents the DNA, and the arrow marks the transcriptional start site. The box marks the response element, either in an open configuration (solid lines) i.e., accessible for factor binding, or in a closed configuration (broken lines), i.e., not accessible for factor binding.

and to direct retrovirus integration to discrete sites where the major groove of the DNA is facing the periphery (Pryciak and Varmus 1992). This demonstrates that nucleosome positioning does influence protein-DNA interactions even at the base-pair level in the living cell.

\section{Materials and methods}

\section{DNA constructs}

Vector pGEM-Q2 was produced from pGEM-2 (Promega) by cutting with restriction enzymes SstI and Sall to remove the unique symmetric $A v a I$ site and cloning the sequence CAGGCCTACTAGTGATACTCGGGATG into the SstI and SalI site to introduce an asymmetric $A v a I$ site. The oligonucleotides TG, Go, and GoA (Fig. 1) were synthesized on a Gene Assembler (Pharmacia) and purified by electrophoresis on $12 \%$ denaturing polyacrylamide gels. Plasmids Go4, 3Go1, 2Go2, 3GoAl, and 2GoA2 were constructed by sequential cloning of TG and Go or GoA into the asymmetric AvaI site of vector pGEM-Q2. The terminology of the constructs gives the number of 20-bp TG motifs and the position in relation to the GRE (Go), for example, $2 \mathrm{Go} 2$ consists of a central GRE flanked by $2 \times 20$ bp of TG motif on both sides. A plasmid lacking GRE but harboring $5 \times 20$ bp of TG motif was used as a negative control and was kindly provided by Drs. Schrader and Crothers (Schrader and Crothers 1989).

\section{Nucleosome reconstitution}

The 165-bp EcoRI-HindIII fragment from 2.5 pmoles of plasmid DNA was 5 '-end-labeled by use of $\mathrm{T} 4$ polynucleotide kinase and $\left[\gamma^{-32} \mathrm{P}\right] \mathrm{ATP}(6000 \mathrm{Ci} / \mathrm{mmole})$. The various EcoRI-HindIII inserts were separated from the vectors on $1 \%$ SeaPlaque gel (FMC Bioproducts) containing $0.5 \mu \mathrm{g} / \mathrm{ml}$ of ethidium bromide, localized by UV light and purified from the SeaPlaque gel with Gelase according to the supplier's recommendations (Epicentre Technologies). In vitro nucleosome reconstitution and purification by glycerol gradient centrifugation was performed as described previously (Perlmann and Wrange 1988). The purified mononucleosome preparations were stored at $4^{\circ} \mathrm{C}$ and showed no dissociation of DNA from the histone octamers even after several weeks of storage.

\section{Nuclease protection}

Exonuclease III (Pharmacia) digestion was performed with 1.5 fmole of DNA and $15 \mathrm{U} / \mathrm{ml}$ of enzyme in $40 \mu \mathrm{l}$ volume and incubated at $25^{\circ} \mathrm{C}$ for $2-16 \mathrm{~min}$. The reactions were stopped by the addition of SDS and $\mathrm{Na}_{2}$ EDTA to final concentrations of $0.125 \%$ (wt/vol) and $15 \mathrm{~mm}$, respectively, followed by analysis on $6 \%$ denaturing polyacrylamide gels. DNase I footprinting was performed as described previously (Perlmann and Wrange 1988).

\section{GR binding and mobility retardation assay}

Purified glucocorticoid receptor was prepared as described (Eriksson and Wrange 1990). It was essential to titrate the optimal concentration of GR for each type of binding experiment for the following reasons: (1) GR concentrations not exceeding the dissociation constant of the cognate binding site were required to evaluate the relative GR affinity; (2) high enough GR concentration was necessary to render a sufficient signal for quantitation; and (3) a low GR concentration was essential to minimize aggregation and nonspecific binding of GR to nucleosomes and free DNA.

To determine the active GR in terms of DNA binding in the GR preparation used in this study, we measured its capacity to bind a 29-bp fragment of naked DNA harboring the upstream MMTV GRE displayed in Figure 1. This DNA segment was added in excess. Quantitation of specific GR-DNA complex was done by glycerol gradient centrifugation as described previously (Wrange et al. 1989; Perlmann et al. 1990) and showed that $26 \%$ of total GR was able to bind DNA. Quantitation of total GR was based on specific binding of tritium-labeled glucocorticoid hormone at the day of purification (Wrange et al. 1989). It is the concentration of active DNA-binding GR that is given as GR concentration for the various experiments in the text. GR was incubated with $\left[{ }^{32} \mathrm{P}\right]$-labeled mononucleosomes for $45 \mathrm{~min}$ at $25^{\circ} \mathrm{C}$ in GR-binding buffer $[20 \mathrm{mM}$ Tris- $\mathrm{HCl} / \mathrm{pH}$ 7.6), $1 \mathrm{mM} \mathrm{Na}{ }_{2}$ EDTA, $10 \%$ (vol/vol) glycerol, $50 \mathrm{~mm} \mathrm{NaCl}, 5$ $\mathrm{mM}$ DTT, $0.1 \mathrm{mg} / \mathrm{ml}$ pork insulin]. The samples were electrophoresed at $14 \mathrm{~V} / \mathrm{cm}$ on $4 \%$ acrylamide gels (acrylamide/ bisacrylamide $40: 0.5$ ) in $0.25 \times$ TBE buffer (Sambrook et al. 1989 ) for $2 \mathrm{hr}$ at $4^{\circ} \mathrm{C}$. The gels were dried and autoradiographed on x-ray film (Fuji). Routinely, final GR concentrations of 3.6 and $1.2 \mathrm{nM}$ were used for CT. DNA competition experiments and nucleosome titration experiments, respectively.

Quantification of gel bands was performed by PhosphorImager analysis, Image Quant v3.0, Fast Scan system (Molecular Dynamics) (Johnston et al. 1990).

\section{Acknowledgments}

We thank T. Shrader and D. Crothers for providing their plasmid TG-5, here referred to as TG. We are indebted to Ulla Björk for excellent technical assistance. We are grateful to S. Belikov, P. Blomquist, U. Lendahl, L. Westin, L. Wieslander, and K. Zaret for critical reading of the manuscript. This work was supported by grants from the Swedish Cancer Foundation (2222-B9309XBC) and M. Bergwalls Foundation.

The publication costs of this article were defrayed in part by payment of page charges. This article must therefore be hereby marked "advertisement" in accordance with 18 USC section 1734 solely to indicate this fact. 


\section{References}

Arents, G., R.W. Burlingame, B.-C. Wang, W.E. Love, and E.N. Moudrianakis. 1991. The nucleosomal core histone octamer at 3.1 Á resolution: A tripartite protein assembly and a lefthanded superhelix. Proc. Natl. Acad. Sci. 88: 10148-10152.

Bresnick, E.H., M. Bustin, V. Marsaud, H. Richard-Foy, and G.L. Hager. 1992. The transcriptionally active MMTV promoter is depleted of histone Hl. Nucleic Acids Res. 20: 273-278.

Carr, K.D. and H. Richard-Foy. 1990. Glucocorticoids locally disrupt an array of positioned nucleosomes on the rat tyrosine aminotransferase promoter in hepatoma cells. Proc. Natl. Acad. Sci. 87: 9300-9304.

Clark, J.D. and A.P. Wolffe. 1991. Superhelical stress and nucleosome-mediated repression of 5S RNA transcription in vitro. EMBO f. 10: 3419-3428.

Cordingley, M.G., A.T. Riegel, and G.L. Hager. 1987. Steroiddependent interaction of transcription factors with the inducible promoter of mouse mammary tumor virus in vivo. Cell 48: 261-270.

Durrin, L.K., R.K. Mann, P.S. Kayne, and M. Grunstein. 1991. Yeast histone $\mathrm{H} 4 \mathrm{~N}$-terminal sequence is required for promoter activation in vivo. Cell 65: 1023-1031.

Durrin, L.K., R.K. Mann, and M. Grunstein. 1992. Nucleosome loss activates CUP1 and HIS3 promoters to fully induced levels in yeast Saccharomyces cerevisiae. Mol. Cell. Biol. 12: 1621-1629.

Eriksson, P., and Ö. Wrange. 1990. Protein-protein contacts in the glucocorticoid receptor homodimer influence its DNA binding properties. J. Biol. Chem, 265: 3535-3542.

Han, M. and M. Grunstein. 1988. Nucleosome loss activates yeast downstream promoters in vivo. Cell 55: 1137-1145.

Hayes, J.J., D.J. Clark, and A.P. Wolffe. 1991. Histone contributions to the structure of DNA in the nucleosome. Proc. Natl. Acad. Sci. 88: 6829-6833.

Hirschhorn, J.N., S.A. Brown, C.D. Clark, and F. Winston. 1992. Evidence that SNF2/SWI2 and SNF5 activate transcription in yeast by altering chromatin structure. Genes \& Dev. 6: 2288-2298.

Hogan, M.E., T.F. Rooney, and R.H. Austin. 1987. Evidence for kinks in DNA folding in the nucleosome. Nature 328: 554 557.

Jantzen, H.-M., U. Strähle, B. Gloss, F. Stewart, W. Schmid, M. Boshart, R. Miksicek, and G. Schütz. 1987. Cooperativity of glucocorticoid response elements located far upstream of the tyrosine aminotransferase gene. Cell 49: 29-38.

Johnson, L.M., P.S. Kayne, E.S. Kahn, and M. Grunstein. 1990. Genetic evidence for an interaction between SIR3 and histone $\mathrm{H} 4$ in the repression of the silent mating loci in Saccharomyces cerevisiae. Proc. Natl. Acad. Sci. 87:62866290.

Johnston, R.F., S.C. Pickett, and D.L. Barker. 1990. Autoradiography using storage phosphor technology. Electrophoresis 11: 355-360.

Knezetic, J.A. and D.S. Luse. 1986. The presence of nucleosomes on a DNA template prevents initiation by RNA polymerase II in vitro. Cell 45: 95-104.

Laybourn, P.J. and J.T. Kadonaga. 1991. Role of nucleosomal cores and histone $\mathrm{H} 1$ in regulation of transcription by RNA polymerase II. Science 254: 238-245.

Lee, D.Y., J.J. Hayes, D. Pruss, and A.P. Wolffe. 1993. A positive role for histone acetylation in transcription factor access to nucleosomal DNA. Cell 72: 73-84.

Lorch, Y., J.W. LaPointe, and R.D. Kornberg. 1987. Nucleosomes inhibit the initiation of transcription but allow chain elongation with the displacement of histones. Cell 49: 203-
210.

Lorch, Y., J.W. LaPointe, and R.D. Kornberg. 1992. Initiation of chromatin templates in a yeast RNA polymerase II transcription system. Genes \& Dev. 6: 2282-2287.

Losa, R. and D.D. Brown. 1987. A bacteriophage RNA polymerase transcribes in vitro through a nucleosome core without displacing it. Cell 50: 801-808.

Luisi, B.F., W.X. Xu, Z. Otwinowski, L.P. Freedman, K.R. Yamamoto, and P.B. Sigler. 1991. Crystallographic analysis of the interaction of the glucocorticoid receptor with DNA. Nature 352: 497-505.

Noll, M. 1974. Internal structure of the chromatin subunit. Nucleic Acids Res. 1: 1573-1578.

Perlmann, T. 1992. Glucocorticoid receptor DNA-binding specificity is increased by the organization of DNA in nucleosomes. Proc. Natl. Acad. Sci. 89: 3884-3888.

Perlmann, T., and Ö. Wrange. 1988. Specific glucocorticoid receptor binding to DNA reconstituted in a nucleosome. EMBO I. 7: 3073-3079.

- 1991. Inhibition of chromatin assembly in Xenopus oocytes correlates with derepression of the mouse mammary tumor virus promoter. Mol. Cell. Biol. 11: 5259-5265.

Perlmann, T., P. Eriksson, and Ö. Wrange. 1990. Quantitative analysis of the glucocorticoid receptor-DNA interaction at the mouse mammary tumor virus glucocorticoid response element. J. Biol. Chem. 265: 17222-17229.

Peterson, C.L. and I. Herskowitz. 1992. Characterization of the yeast SWI1, SWI2, and SWI3 genes, which encode a global activator of transcription. Cell 68: 573-583.

Pina, B., U. Bruggemeier, and M. Beato. 1990. Nucleosome positioning modulates accessibility of regulatory proteins to the mouse mammary tumor virus promoter. Cell 60: 719731.

Pryciak, P.M. and H.E. Varmus. 1992. Nucleosomes, DNAbinding proteins, and DNA sequence modulate retroviral intergration target site selection. Cell 69: 769-780.

Ramsay, N. 1986. Deletion analysis of a DNA sequence that positions itself precisely on the nucleosome core. I. Mol. Biol. 189: 179-188.

Reik, A., G. Schütz, and A.F. Stewart. 1991. Glucocorticoids are required for establishment and maintenance of an alteration in chromatin structure: Induction leads to a reversible disruption of nucleosomes over an enhancer. EMBO $/$. 10: 2569-2576.

Rhodes, D. 1985. Structural analysis of a triple complex between the histone octamer, a Xenopus gene for 5S RNA and transcription factor IIIA. EMBO I. 4: 3473-3482.

Richard-Foy, H. and G.L. Hager. 1987. Sequence-specific positioning of nucleosomes over the steroid-inducible MMTV promoter. $E M B O$ I. 6: 2321-2328.

Richmond, T.J., J.T. Finch, B. Rushton, D. Rhodes, and A. Klug. 1984. Structure of the nucleosome core particle at $7^{\prime}$ resolution. Nature 311: 532-537.

Sambrook, J., E.F. Fritsch, and T. Maniatis. 1989. Molecular cloning: A laboratory manual, 2nd ed. Cold Spring Harbor Laboratory Press, Cold Spring Harbor, New York.

Scheidereit, C. and M. Beato. 1984. Contacts between hormone receptor and DNA double helix within a glucocortocoid regulatory element of mouse mammary tumor virus. Proc. Natl. Acad. Sci. 81: 3029-3033.

Shrader, T.E. and D.M. Crothers. 1989. Artificial nucleosome positioning sequences. Proc. Natl. Acad. Sci. 86: 7418-7422.

- 1990. Effects of DNA sequence and histone-histone interactions on nucleosome placement. I. Mol. Biol. 216: 6984.

Simpson, R.T. 1990. Nucleosome positioning can affect the 


\section{Li and Wrange}

function of a cis-acting DNA element in vivo. Nature 343: $387-389$.

. 1991. Nucleosome positioning: occurrence, mechanisms and functional consequences. Prog. Nucleic Acids Res. Mol. Biol. 40: 143-184.

Straka, C. and W. Hörz. 1991. A functional role for nucleosomes in the repression of a yeast promoter. $E M B O J .10: 361-368$. Taylor, I.C.A., J.L. Workman, T.J. Schuetz, and R.E. Kingston. 1991. Facilitated binding of GAL4 and heat shock factor to nucleosomal templates: Differential function of DNA-binding domains. Genes \& Dev. 5: 1285-1298.

Tsai, S.Y., J. Carlstedt-Duke, N.L. Weigel, K. Dahlman, J.-A. Gustafsson, M.-J. Tsai, and B.W. O'Malley. 1988. Molecular interactions of steroid hormone receptor with its enhancer element: Evidence for receptor dimer formation. Cell 55: 361-369.

Winston, F. and M. Carlson. 1992. Yeast SNF/SWI transcriptional activators and the STP/SIN chromatin connection. Trends Genet. 8: 387-391.

Workman, J.L., S.M. Abmayr, W.A. Cromlish, and R.G. Roeder. 1988. Transcriptional regulation by the immediate early protein of Pseudorabies virus during in vitro nucleosome assembly. Cell 55: 211-219.

Wrange, Ö., P. Eriksson, and T. Perlmann. 1989. The purified activated glucocorticoid receptor is a homodimer. J. Biol. Chem. 264: 5253-5259.

Yoshinaga, S.K., C.L. Peterson, I. Herskowitz, and K.R. Yamamoto. 1992. Role of SWI1, SWI2, and SWI3 proteins for transcriptional enhancement by steroid receptor. Science 258: 1598-1604.

Zaret, K.S. and K.R. Yamamoto. 1984. Reversible and persistent changes in chromatin structure accompany activation of a glucocorticoid-dependent enhancer element. Cell 38: 29-38. 


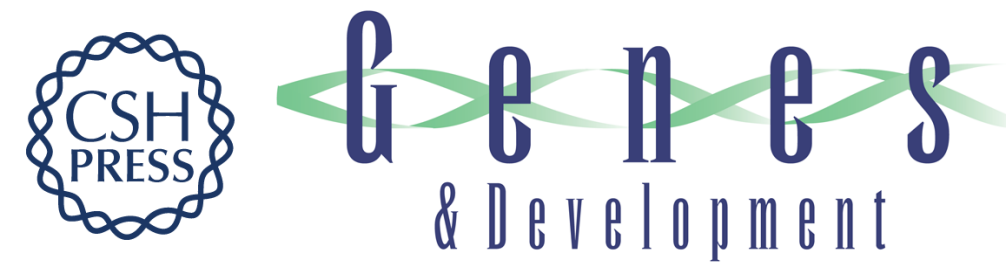

\section{Translational positioning of a nucleosomal glucocorticoid response element modulates glucocorticoid receptor affinity.}

Q Li and O Wrange

Genes Dev. 1993, 7:

Access the most recent version at doi:10.1101/gad.7.12a.2471

References

This article cites 48 articles, 17 of which can be accessed free at:

http://genesdev.cshlp.org/content/7/12a/2471.full.html\#ref-list-1

License

Email Alerting

Receive free email alerts when new articles cite this article - sign up in the box at the top Service right corner of the article or click here.

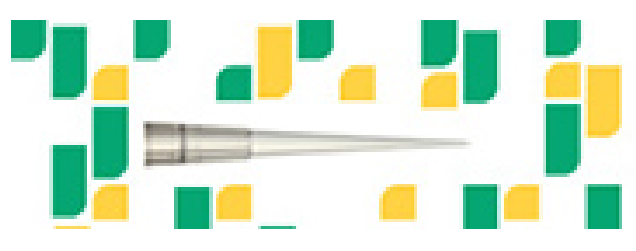

Focused on your science. 